\section{Prevalence of antibiotic resistance and blalMP-1 gene among Pseudomonas aerugi- nosa strains isolated from burn and urinary tract infections in Isfahan, central Iran}

Naeimeh Sadat Hashemi, ${ }^{1}$

Meysam Mojiri, ${ }^{1}$ Parivash Yazdani

Kachouyi, ${ }^{2}$ Shiva Eskandari, ${ }^{1}$

Mehrsa Mohammadian, ${ }^{3}$ Iman Alavi ${ }^{1}$

${ }^{1}$ Faculty of Basic Sciences, Shahrekord Branch, Islamic Azad University, Shahrekord; ${ }^{2}$ Department of Emergency Medicine, Ahvaz Jundishapur University of Medical Sciences, Ahvaz; ${ }^{3}$ Faculty of Biology, Falavarjan Branch, Islamic Azad University, Falavarjan, Iran

\section{Abstract \\ Pseudomonas aeruginosa is one of the} most important opportunistic pathogens responsible for various types of hospital infections. High prevalence of antibiotic resistance in $P$. aeruginosa strains of human clinical samples cause more severe diseases for a longer period of time. The current research was done in order to study the distribution of blaIMP-1 gene among the imipenem-resistant $P$. aeruginosa strains isolated from burn and urinary tract infections of hospitalized patients. Two-hundred and forty-three $P$. aeruginosa isolates recovered from the cases of burn and urinary tract infections of inpatients and outpatients were analysis for antibiotic resistance pattern using the disk diffusion method. Then, imipenem-resistant isolates were further analyzed for distribution of blaIMP-1 gene using the PCR. Of 243 P. aeruginosa isolates, 146 strains $(60.08 \%)$ were taken from outpatients and 97 strains (39.91\%) were taken from inpatients. $P$. aeruginosa isolates harbored the highest levels of resistance against streptomycin (100\%), nalidixic acid $(100 \%)$, aztreonam $(100 \%)$, cotrimoxazole $(95.47 \%)$, ciprofloxacin $(88.47 \%)$, cefotaxime $(84.36 \%)$ and gentamycin $(83.95 \%)$. Inpatients had a relatively higher levels of antibiotic resistance. One-hundred and twenty-one out of 126 (96.03\%) imipenem-resistant $P$. aeruginosa isolates harbored the blaIMP-1 gene. Inpatients also had a relatively higher prevalence of blaIMP-1 gene. High prevalence of blaIMP-1 gene and also imipenemresistant $P$. aeruginosa are important public health issue. Clinical laboratories should consider the detection of the blaIMP-1 gene among the $P$. aeruginosa isolates of clinical samples.

\section{Introduction}

Superficial infections and especially burn infections (BIs) are important cause of emergency health care associated problems al-around the world. Superficial infections caused longer hospital stays, more expensive hospitalizations, and increased mortality. ${ }^{1}$ The annual superficial infection care products market is projected to reach $\$ 15.5$ billion by $2010 .{ }^{1}$ Urinary tract infections (UTIs) are one of the most common bacterial infections diseases in human. ${ }^{2-4}$ UTIs account for more than 8 million referrals to hospitals, 1.5 million hospitalizations, and 300,000 severe clinical syndromes in the United States annually. ${ }^{2-4}$ UTIs is an important cause of mortality and morbidity alaround the world. ${ }^{5,6}$

Pseudomonas aeruginosa ( $P$. aerugi$n o s a$ ) is a non-fermentative, aerobic, Gramnegative rod shape bacterium and is responsible for severe human clinical infections such as BIs, UTIs, pneumonia, wound, reproductive tract, respiratory tract and superficial and gastrointestinal infections, cystic fibrosis, ecthyma gangrenosum and black necrotic lesions. ${ }^{7}$ It is also related to sever cases of hospital-acquired and healthcare associated infections globally. ${ }^{?}$

Resistant $P$. aeruginosa strains cause more severe clinical diseases which are mainly difficult to treatment with routine antibiotics. ${ }^{8,9}$ Treatment of UTIs and BIs caused by this bacterium is often started empirically and therapy is based on information determined from the antimicrobial resistance pattern. ${ }^{8,9}$ However, a large proportion of uncontrolled antibiotic usage has subsidized to the development of resistance in $P$. aeruginosa strains. ${ }^{8,9} P$. aeruginosa strains exhibits the highest levels of resistance against fluoroquinolones, beta-lactams, penicilins, tetracyclines, carbapenems, aminoglycosides, macrolides and other types of antimicrobial agents. High levels of antibiotic resistance in the $P$. aeruginosa isolates of UTIs and BIs have been reported previously. ${ }^{8,9}$ Recently, clinical isolates of the $P$. aeruginosa exhibited the high levels of resistance against imipenem antibiotic agent. ${ }^{10}$ Documented data revealed that blaIMP gene is the most prevalent antibiotic resistance marker in the clinical isolates of imipenem-resistant $P$. aeruginosa. ${ }^{10}$

According to the high clinical standing of $P$. aeruginosa in hospital infections and
Correspondence: Iman Alavi, Department of Microbiology, Faculty of Basic Sciences, Shahrekord Branch, Islamic Azad University, Shahrekord, Iran.

Tel.: +98.913.7004627.

E-mail: alaviiman66@gmail.com

Key world: Pseudomonas aeruginosa, Antibiotic resistance, hospital infections, Imipenem-resistant, IMP-1 gene.

Acknowledgments: Authors would like to thanks from the staffs of the Biotechnology Research Center of the Islamic Azad University of Shahrekord for their clinical supports. This work was supported by the Islamic Azad University, Shahrekord Branch, Shahrekord, Iran.

Contributions: the authors contributed equally

Conflict of interest: the authors declare no potential conflict of interest.

Received for publication: 2 July 2017

Accepted for publication: 31 July 2017.

This work is licensed under a Creative Commons Attribution NonCommercial 4.0 License (CC BY-NC 4.0).

(C) Copyright N.S. Hashemi et al., 2017 Licensee PAGEPress, Italy

Microbiology Research 2017; 8:7295

doi:10.4081/mr.2017.7295

also lack of microbiological and epidemiological investigations about the antibiotic resistance and also distribution of blaIMP gene, the current research was done to study the antibiotic resistance pattern of $P$. aeruginosa isolates of BI and UTIs and study the distribution of blaIMP-1 gene among the imipenem-resistant strains.

\section{Materials and Methods}

\section{Ethical considerations}

Ethical committees of the Alzahra and Kashani Hospitals were approved the general principles and framework of the current investigation. Written informed consent was obtained from all of the study patients or their parents. Personal information of all patients were remained secret.

\section{Bacterial strains}

From March 2013 to June 2014, a total of 243 P. aeruginosa isolates were referred to the Microbiology Research Center of the Islamic Azad University of Shahrekord, Iran. Isolates were recovered from impa- 
tient and outpatients of the Kashani and Alzahra Hospitals, Isfahan, Iran. P. aeruginosa strains were isolated from the cases of BIs $(\mathrm{n}=170)$ and UTIs $(\mathrm{n}=73)$. All of the $P$. aeruginosa isolates were further identified according to the Gram staining, oxidase test, citrate utilization test, motility test, urease production test, gelatinase liquefaction, catalase test, triple sugar iron (TSI) agar test, oxidative-fermentative test, alkaline protease production, nitrate reduction test, indole test, haemolysin production and lecithinase production.

\section{Antibiotic susceptibility test}

Pattern of antimicrobial resistance was studied using the simple disk diffusion technique. The Mueller-Hinton agar (Merck, Germany) medium was used for this purpose. Antibiotic resistance of $P$. aeruginosa strains against 22 commonly used antibiotics in the cases of UTIs was determined using the instruction of Clinical and Laboratory Standards Institute guidelines. ${ }^{11}$ Susceptibility of $P$. aeruginosa strains were tested against gentamycin $(10 \mu \mathrm{g} /$ disk $)$, imipenem (30 u/disk), cefotaxime (30 $\mu \mathrm{g} /$ disk), ciprofloxacin (5 $\mu \mathrm{g} /$ disk), cotrimoxazole (30 $\mu \mathrm{g} /$ disk), ceftazidime (30 $\mu \mathrm{g} /$ disk), chloramphenicol (30 $\mu \mathrm{g} /$ disk), streptomycin (10 $\mu \mathrm{g} /$ disk), nalidixic acid (30 $\mu \mathrm{g} /$ disk) and aztreonam $(30 \mu \mathrm{g} /$ disk $)$ antibiotic agents (Oxoid, UK). All of the inoculated plates were aerobically incubated at $37^{\circ} \mathrm{C}$ for $18-24 \mathrm{~h}$ in an aerobic atmosphere. Results were interpreted based on the instruction provided by CLSI (2012). ${ }^{11}$ In all reactions, the $P$. aeruginosa (ATCC 27853) was used as quality control organisms.

\section{DNA extraction}

Total genomic DNA was extracted from the bacterial colonies. A single colony was inoculated on $5 \mathrm{~mL}$ of brain heart infusion broth and incubated over night at $37^{\circ} \mathrm{C}$. Then $1.5 \mathrm{~mL}$ of a saturated culture was harvested with centrifugation for $5 \mathrm{~min}$. at $14,000 \mathrm{rpm}$. The cell pellet was resuspended and lysed in $200 \mu 1$ of lysis buffer (40 $\mathrm{mM}$ Tris-acetate $\mathrm{pH} 7.8,20 \mathrm{mM}$ sodiumacetate, $1 \mathrm{mM}$ EDTA, $1 \%$ SDS) by vigorous pipetting. To remove most proteins and cell debris, $66 \mu \mathrm{L}$ of $5 \mathrm{M} \mathrm{NaCl}$ solution was added and mixed well, and then the viscous mixture was centrifuged at $12,000 \mathrm{rpm}$ for $10 \mathrm{~min}$. at $4^{\circ} \mathrm{C}$. After transferring the clear supernatant into a new Eppendorf tube, an equal volume of chloroform was added, and the tube was gently inverted at least 50 times when a milky solution was completely formed. Following centrifugation at $14,000 \mathrm{rpm}$ for $5 \mathrm{~min}$, the supernatant is then removed to another eppendorf tube and double volume of $100 \%$ ethanol was added. The tubes were inverted 5 to 6 times gently, then centrifuged at $10,000 \mathrm{rpm}$ for 5 minutes. The supernatant was discarded and $1 \mathrm{~mL}$ of ethanol $(70 \%)$ was added to the pellet, and tubes centrifuged at $10,000 \mathrm{rpm}$ for $5 \mathrm{~min}$ utes. Finally, the supernatant discarded and the pellet was dried for $10 \mathrm{~min}$ at room temperature, the pellet was resuspended by 100 $\mu \mathrm{L} \mathrm{H} 2 \mathrm{O}$. The stock was kept at $-20^{\circ} \mathrm{C}$ until use.

\section{PCR amplification of blaIMP-1 gene}

Imipenem-resistant $P$ aeruginosa strains were subjected to PCR amplification of the blaIMP-1 gene. The PCR mixture contained $200 \mu \mathrm{M}$ of each dNTP (Fermentas, Germany), PCR buffer (10 mM Tris/ $\mathrm{HCl}, 50 \mathrm{mM} \mathrm{KCl}, 1.5 \mathrm{mM} \mathrm{MgCl} 2, \mathrm{pH}$ 8.3), DMSO at a final concentration of $4 \%$, 12.5 pmol of each primer (blaIMP-1; F: 5' ACCGCAGCAGAGTCTTTGCC-3' and blaIMP-1; R: 5'-ACAACCAGTTTTGCCTTACC-3' (587 bp), ${ }^{12} 1$ U Taq DNA polymerase (Fermentas, Germany) and 25 ng DNA template. The DNA was amplified in a programmable thermal cycler (Eppendorf, Mastercycler ${ }^{\circledR} \quad 5330$, Eppendorf-Netheler-Hinz $\mathrm{GmbH}$, Hamburg, Germany) PCR device using the following protocol: $95^{\circ} \mathrm{C}$ for $5 \mathrm{~min}, 30$ cycles of $94^{\circ} \mathrm{C}$ for $30 \mathrm{~s}, 55^{\circ} \mathrm{C}$ for $30 \mathrm{~s}, 72^{\circ} \mathrm{C}$ for $30 \mathrm{~s}$, and $72^{\circ} \mathrm{C}$ for $5 \mathrm{~min}$. P. aeruginosa ATCC 27853 were used as positive controls and PCR grade water Fermentas, Germany) was used as a negative control in all PCR reactions. Ten microliters of PCR products were resolved on a $1 \%$ agarose gel containing $0.5 \mathrm{mg} / \mathrm{ml}$ of ethidium bromide in Trisborate-EDTA buffer at $90 \mathrm{~V}$ for $30 \mathrm{~min}$, also using suitable molecular weight markers (100 bp, Fermentas, Germany). The products were examined under ultraviolet illumination.

\section{Statistical analysis}

The results were transferred to a Microsoft Excel spreadsheet (Microsoft Corp., Redmond, WA) for analysis. Statistical analysis was performed using SPSS/21.0 software (SPSS Inc., Chicago, IL) for significant relationship between pattern of antibiotic resistance and also distribution of the blaIMP-1 gene of P. aeruginosa isolates. The chi-square test and Fisher's exact 2-tailed test analysis were performed in this study. Statistical significance was regarded at a $\mathrm{P}<0.05$.

\section{Results}

Among all 243 isolates of $P$. aeruginosa, 146 strains $(60.08 \%)$ were taken from outpatients, while 97 strains (39.91\%) were taken from inpatients. Table 1 represents the results of the disk diffusion method for the $P$. aeruginosa strains isolated from human clinical samples. $P$. aeruginosa strains of our investigation harbored the highest levels of resistance against streptomycin $(100 \%)$, nalidixic acid $(100 \%)$, aztreonam (100\%), cotrimoxazole $(95.47 \%)$, ciprofloxacin $(88.47 \%)$, cefotaxime $(84.36 \%)$ and gentamycin (83.95\%). P. aeruginosa strains of samples taken from outpatients had the highest levels of resistance against

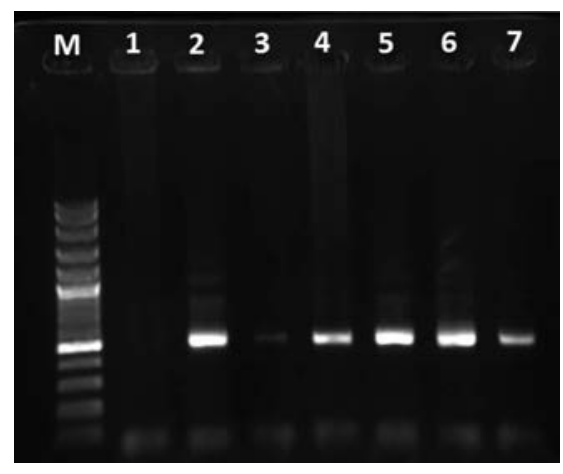

Figure 1. Results of the gel electrophoresis for amplification of the blaIMP-1 gene among studied $P$. aeruginosa isolates. M: 100 bp ladder (Fermentas, Germany), 1: Negative control, 2: Positive control and 37: Positive samples for the blaIMP-1 gene (587 bp).

Table 1. Antibiotic resistance pattern of the P. aeruginosa strains isolated from human clinical infections.

\begin{tabular}{|c|c|c|c|c|c|c|c|c|c|c|}
\hline \multirow{2}{*}{$\begin{array}{l}\text { Clinical infections } \\
\text { (N. P. aeruginosa) }\end{array}$} & \multicolumn{10}{|c|}{ Antibiotic resistance pattern (\%) } \\
\hline & G10 & Imp & Cef & Cip & Cot & Cfz & C30 & S10 & Nlx & Az \\
\hline Outpatients (146) & $114(78.08)$ & $74(50.68)$ & $115(78.77)$ & $124(84.93)$ & $137(93.83)$ & $105(71.92)$ & $107(73.29)$ & $146(100)$ & $146(100)$ & $146(100)$ \\
\hline Inpatients (97) & $90(92.78)$ & $52(53.61)$ & $90(92.78)$ & $91(93.81)$ & $95(97.94)$ & $83(85.57)$ & $85(87.63)$ & $97(100)$ & $97(100)$ & $97(100)$ \\
\hline Total (243) & $204(83.95)$ & $126(51.85)$ & $205(84.36)$ & $215(88.47)$ & $232(95.47)$ & $188(77.36)$ & $192(79.01)$ & $243(100)$ & $243(100)$ & $243(100)$ \\
\hline
\end{tabular}


streptomycin (100\%), nalidixic acid $(100 \%)$, aztreonam $(100 \%)$, cotrimoxazole $(93.83 \%)$ and ciprofloxacin (84.93\%), while those of inpatients had the highest against streptomycin (100\%), nalidixic acid (100\%), aztreonam $(100 \%)$, cotrimoxazole (97.94\%), ciprofloxacin (93.81\%), cefotaxime $(92.78 \%)$ and gentamycin $(92.78 \%)$. There were no statistically significant differences for the prevalence of antibiotic resistance between types of samples. Totally, $51.85 \%$ of $P$. aeruginosa strains had resistance against imipenem.

Table 2 represents the distribution of blaIMP-1 gene among the imipenem resistant $P$. aeruginosa strains isolated from human clinical infections. Prevalence of the blaIMP-1 gene among the imipenem-resistant $P$. aeruginosa strains recovered from outpatients and inpatients were $95.94 \%$ and $96.15 \%$, respectively (Figure 1).

\section{Discussion}

The present investigation focused on the antibiotic resistance pattern of the $P$. aeruginosa strains of BIs and UTIs. As it showed, $P$. aeruginosa had significant impact in the occurrence of BIs and UTIs. High impact of $P$. aeruginosa in hospital infections has been reported from other sites of the world including Brazil, ${ }^{13}$ Italy, ${ }^{14}$ Germany, ${ }^{15}$ United States ${ }^{16}$ and South Africa. ${ }^{17}$ We also found that P. aeruginosa strains harbored the high levels of resistance against commonly used antibiotic and especially streptomycin, nalidixic acid, aztreonam, cotrimoxazole, ciprofloxacin, cefotaxime and gentamycin. Of studies which were conducted in this field, ${ }^{18-23}$ all have shown a high distribution of antibiotic resistance against ampicillin, gentamycin, cotrimoxazole, ciprofloxacin, cefotaxime and amikacin. Presence of considerable levels of resistance against imipenem have been reported previously from Iran, ${ }^{19,20}$ Turkey, ${ }^{24}$ India ${ }^{25}$ and Indonesia. ${ }^{26}$ Onguru et al. $(2008)^{27}$ reported that the $P$. aeruginosa strains of various clinical sources were resistant to imipenem (44.1\%) which was entirely high. They showed that imipenem resistant strains were also resistant to amikacin (70\%), gentamycin $(85 \%)$, tobramycin $(87 \%)$, cefepime $(81 \%)$, piperacillin (61\%) and ciprofloxacin (77\%). Our results revealed that $51.85 \%$ of $P$. aeruginosa strains were resistant to imipenem. Indiscriminate, unauthorized and illegal prescription on antibiotic agents and especially imipenem are the main factors causing high prevalence of resistance of $P$. aeruginosa strains.

Shahini et al. $(2012)^{28}$ reported that $P$. aeruginosa strains of Iranian cases of infections had the highest levels of resistance amoxicillin $(100 \%)$, cefepime $(52 \%)$, trimethoprim $(100 \%)$, imipenem $(60 \%)$, tetracycline $(100 \%)$, clavunic acid $(69.2 \%)$, ampicillin $(100 \%)$, carbenicillin $(90 \%)$, gentamycin $(50 \%)$, ticarcillin $(100 \%)$, ceftazidime (80\%), and ciprofloxacin (40\%). Fazeli and Momtaz (2014) ${ }^{29}$ reported more similar results with our findings. They showed that $P$. aeruginosa strains of clinical infections harbored the highest levels of resistance against penicillin $(100 \%)$, tetracycline $(90.19 \%)$, streptomycin $(64.70 \%)$ and erythromycin $(43.13 \%)$. Shiny et al. $(2013)^{30}$ reported that $P$. aeruginosa strains of pus and urine samples had the highest levels of resistance against imipenem (100\%) and cefotaxime (93.75\%). Akingbade et al. $(2012)^{31}$ reported that $P$. aeruginosa isolates of infectious samples harbored considerable levels of resistance against tetracycline $(70.9 \%)$, amoxicillin $(92.7 \%)$, erythromycin $(72.7 \%)$, ampicillin (90\%), cotrimoxazole $(77.3 \%)$, cloxacillin $(88.2 \%)$, ofloxacin $(690 \%)$ and streptomycin (65.5\%). Availability of antibiotic agents, cost of them and idea of medical practitioners for prescription of antibiotics are the main factors caused differences in the prevalence of antibiotic resistance in various studies.

The most actual antibiotics that can be used against $P$. aeruginosa infections are beta-lactam antibiotics in which imipenem as a carbapenem is considered as the most suitable antibiotic. However, carbapenem resistance occurs because of decrease in antibiotics absorption due to lack of an outer membrane porin, exclusion from the cell by efflux pump, decrease in outer membrane permeability and production of metallo-beta-lactamases (MBL). The IMP type is the most clinically significant carbapenemases which encoded by blaIMP gene and harden all treatments done using the imipenem. ${ }^{31}$ Our results also showed that about $96.03 \%$ of imipenem-resistant $P$. aeruginosa strains harbored the blaIMP-1 gene which was considerable. Peymani et al. $(2015)^{32}$ revealed that 107 (35.66\%) P. aeruginosa isolates were non-susceptible to imipenem and/or meropenem and among them, $56(52.3 \%)$ isolates were MBL producer. Twenty-four isolates of 56 (42.85\%) MBL producer strains harbored MBLencoding genes. Prevalence of blaIMP-1 gene was $25 \%$ which also was lower than our findings. Moosavian et al. $(2015)^{33}$ reported that of 236 examined $P$. aeruginosa isolates, 122 isolates $(51.40 \%)$ were resistant to imipenem. They showed that 67 strains $(55 \%)$ of imipenem-resistant $P$. aeruginosa isolates harbored blaIMP-1 gene which was lower than our findings. Abiri et al. $(2015)^{34}$ reported that among the 225 P. aeruginosa isolates, $33.7 \%(76 / 225)$ and $18.1 \%(41 / 225)$ were resistant to imipenem and meropenem, respectively. They showed that of the 76 imipenem-resistant $P$. aeruginosa strains, $45(59.2 \%)$ were positive for MBLs and 34 (75\%) strains carried the blaIMP-1 gene which was similar to our findings. Tarashi et al. (2016) ${ }^{35}$ indicated that among 278 imipenem-resistant $P$. aeruginosa strains, 178 (64.02\%) were MBL producers. The blaIMP-1 gene was detected in $16.8 \%$ of $P$. aeruginosa isolates. As far as we know, the present study reported the highest prevalence of blaIMP-1 gene among the imipenem-resistant $P$. aeruginosa strains of human clinical samples. Differences in types of samples is the main factor for various prevalence rate of blaIMP-1 gene reported in different studies.

\section{Conclusions}

In conclusion, we identified a large number of $P$. aeruginosa strains in the BIs and UTIs samples taken from inpatients and outpatients. Resistance against streptomycin, nalidixic acid, aztreonam, cotrimoxazole, ciprofloxacin, cefotaxime and gentamycin was considerable in the $P$. aeruginosa strains of clinical samples. Moderate prevalence of resistance against imipenem was supported by the high prevalence of blaIMP gene. High prevalence of this gene poses an important public health threat regarding the occurrence of resistance against imipenem in the clinical isolates of $P$. aeruginosa. It seems that prescription of imipenem is not effective for treatment of the cases of $P$. aeruginosa infection.

Table 2. Prevalence of blaIMP-1 gene among the imipenem resistant P. aeruginosa strains isolated from human clinical infections.

\begin{tabular}{lc} 
Samples (No. imipenem-resistant isolates) & Distribution of blalMP-1 gene (\%) \\
Outpatients (74) & $71(95.94)$ \\
Inpatients (52) & $50(96.15)$ \\
\hline Total (126) & $121(96.03)$ \\
\hline
\end{tabular}


However, additional investigations should perform to found other epidemiological aspects of the MBL producing $P$. aeruginosa, detection of blaIMP-1 gene recommended to be important in all clinical microbiology laboratories.

\section{References}

1. Sen CK, Gordillo GM, Roy S, et al. Human skin wounds: a major and snowballing threat to public health and the economy. Wound Repair Regen 2009;17:763-71.

2. Mittal R, Aggarwal S, Sharma S, et al. Urinary tract infections caused by Pseudomonas aeruginosa: a minireview. J Infect Public Health 2009;2:101-11.

3. Kucheria R, Dasgupta P, Sacks SH, et al. Urinary tract infections: new insights into a common problem. Postgrad Med J 2005;81:83-6.

4. Shaikh N, Morone NE, Bost JE, et al. Prevalence of urinary tract infection in childhood: a meta-analysis. Pediatr Infect Dis J 2008;27:302-8.

5. Sobczyk D, Krynicki T, Blumczyński A, et al. New, successful treatment of urinary tract infection caused by Pseudomonas aeruginosa. Przegl Lek 2006;63:140-1.

6. Dormanesh B, Safarpoor Dehkordi F, Hosseini S, et al. Virulence factors and O-serogroups profiles of uropathogenic Escherichia coli isolated from Iranian pediatric patients. Iran Red Crescent Med J 2014;16:e14627.

7. Bodey GP, Bolivar R, Fainstein V, et al. Infections caused by Pseudomonas aeruginosa. Rev Infect Dis 1983;5:279313.

8. Jeukens J, Freschi L, Kukavica-Ibrulj I, et al. Genomics of antibiotic-resistance prediction in Pseudomonas aeruginosa. Ann N Y Acad Sci. 2017.

9. Lister PD, Wolter DJ, Hanson ND. Antibacterial-resistant Pseudomonas aeruginosa: clinical impact and complex regulation of chromosomally encoded resistance mechanisms. Clin Microbiol Rev 2009;22:582-610.

10. Sedighi M, Salehi-Abargouei A, Oryan G, ET AL. Epidemiology of VIM-1imipenem resistant Pseudomonas aeruginosa in Iran: a systematic review and meta-analysis. J Res Med Sci 2014;19:899-903.

11. Clinical and Laboratory Standards Institute (CLSI). Performance standards for antimicrobial susceptibility testing; twenty-second informational supplement. M100-S21. Wayne Pa: CLSI;2012.
12. Ghamgosha M, Shahrekizahedani S, Kafilzadeh F, et al. Metallo-betaLactamase VIM-1, SPM-1, and IMP-1 genes among clinical Pseudomonas aeruginosa species isolated in Zahedan, Iran. Jundishapur J Microbiol 2015;8:e17489.

13. Zavascki AP, Gaspareto PB, Martins $\mathrm{AF}$, et al. Outbreak of carbapenemresistant Pseudomonas aeruginosa producing SPM-1 metallo- $\{$ beta $\}$-lactamase in a teaching hospital in southern Brazil. J Antimicrob Chemother 2005;56:1148-51.

14. Lanini S, D'Arezzo S, Puro V, et al. Molecular epidemiology of a Pseudomonas aeruginosa hospital outbreak driven by a contaminated disinfectant-soap dispenser. Plos One 2011;6:e17064.

15. Pitten FA, Panzig B, Schröder G, et al. Transmission of a multiresistant Pseudomonas aeruginosa strain at a German university hospital. J Hosp Infect 2001;47:125-30.

16. Hirsch EB, Tam VH. Impact of multidrug-resistant Pseudomonas aeruginosa infection on patient outcomes. Expert Rev Pharmacoecon Outcomes Res. 2010;10:441-51.

17. Mudau M, Jacobson R, Minenza N, et al. Outbreak of multi-drug resistant Pseudomonas aeruginosa bloodstream infection in the haematology unit of a South African academic hospital. Plos One 2013;8:e55985.

18. Narten M, Rosin N, Schobert M, et al. Susceptibility of Pseudomonas aeruginosa urinary tract isolates and influence of urinary tract conditions on antibiotic tolerance. Curr Microbiol 2012;64:716.

19. Haghi-Ashteiani M, Sadeghifard N, Abedini M, et al. Etiology and antibacterial resistance of bacterial urinary tract infections in children's medical center, Tehran, Iran. Acta Medica Iranica 2007;45:153-7.

20. Mirsoleymani SR, Salimi M, Shareghi Brojeni M, et al. Bacterial pathogens and antimicrobial resistance patterns in pediatric urinary tract infections: a fouryear surveillance study (2009-2012). Int J Pediatr 2014;2014:1-6.

21. Takeyama K, Kunishima Y, Matsukawa $\mathrm{M}$, et al. Multidrug-resistant Pseudomonas aeruginosa isolated from the urine of patients with urinary tract infection. $\mathrm{J}$ Infect Chemother 2002;8:59-63.

22. Jombo GTA, Jonah P, Ayeni JA. Multidrug resistant pseudomonas aeruginosa in contemporary medical practice: findings from urinary isolates at a nigerian university teaching hospital. Niger J Physiol Sci 2008;23:105-9.

23. Chikwendu CI, Amadi ES, Obi RK. Prevalence and antimicrobial resistance in Pseudomonas aeruginosa and Klebsiella pneumoniae isolates from non-clinical urine samples. NY Sci J 2010;3:194-200.

24. Gençer S, Ak Ö, Benzonana N, et al. Susceptibility patterns and cross resistances of antibiotics against Pseudomonas aeruginosa in a teaching hospital of Turkey. Ann Clin Microbiol Antimicrob 2002;1:1-4.

25. Khajuria A, Praharaj AK, Kumar M, et al. Emergence of NDM-1 in the clinical isolates of Pseudomonas aeruginosa in India. J Clin Diagn Res 2013;7:132831.

26. Moehario LH, Hartono TS, Wardoyo $\mathrm{EH}$, et al. Trend of antibiotics susceptibility of multidrugs resistance Pseudomonas aeruginosa in Jakarta and surrounding areas from 2004 to 2010. Afr J Microbiol Res 2012;6:2222-9.

27. Onguru P, Erbay A, Bodur H, et al. Imipenem-resistant Pseudomonas aeruginosa: Risk factors for nosocomial Infections. J Korean Med Sci 2008;23:982-7.

28. Shahini N, Shahini N, Ala S. Determining of resistance and sensitivity of Pseudomonas aeruginosa in Iran in 2010-2011. Res Pharm Sci 2012;7:S884.

29. Fazeli N, Momtaz H. Virulence gene profiles of multidrug-resistant Pseudomonas aeruginosa isolated from Iranian hospital infections. Iran Red Crescent Med J 2014;16:e15722.

30. Shiny PA, Rajendran S, Sarayu YL. A comparative analysis of isolation and antibiotic sensitivity pattern of Pseudomonas aeruginosa isolated from pus and urine with special reference to phenotypic and genotypic expression of extended spectrum beta lactamases (ESBLs). J Acad Clin Microbiol 2013;15:3-6.

31. Akingbade OA, Balogun SA, Ojo DA, et al. Plasmid profile analysis of multidrug resistant Pseudomonas aeruginosa isolated from wound infections in South West, Nigeria. World Appl Sci J 2012;20:766-75.

32. Peymani A, Naserpour Farivar T, Mohammadi Ghanbarlou $M$. Dissemination of Pseudomonas aeruginosa producing bla IMP-1 and bla VIM1 in Qazvin and Alborz educational hospitals, Iran. Iran J Microbiol 2015;7:302-9.

33. Moosavian M, Rahimzadeh M. Molecular detection of metallo- $\beta$-lacta- 
mase genes, blaIMP-1, blaVIM-2 and blaSPM-1 in imipenem resistant Pseudomonas aeruginosa isolated from clinical specimens in teaching hospitals of Ahvaz, Iran. Iran J Microbiol 2016;7:2-6.

34. Abiri R, Mohammadi P, Shavani N, et al. Detection and genetic characterization of metallo- $\beta$-lactamase imp- 1 and vim-2 in Pseudomonas aeruginosa strains from different hospitals in Kermanshah, Iran. Jundishapur J Microbiol 2015;8:e22582.

35. Tarashi S, Goudarzi H, Erfanimanesh S, et al. Phenotypic and molecular detection of Metallo-beta-lactamase genes among imipenem resistant Pseudomonas aeruginosa and Acinetobacter baumannii strains isolated from patients with burn injuries. Arch Clin Infect Dis 2016;11:e39036 